\title{
SYNTHESIS AND CHARACTERIZARTION OF MAGNETIC NANOPARTICLES OF FeCO WITH CORE-SHELL STRUCTURE PRODUCED BY SOL-GEL-PROTEIC METHOD
}

\author{
Dias, D. F. ${ }^{1}, *$; de Sousa, M. F. ${ }^{1}$ Sasaki, J. M. ${ }^{1}$; Braga, T. B. ${ }^{2}$ \\ ${ }^{1}$ Universidade Federal do Ceará. Departamento de Física, Fortaleza, Ceará, Brazil \\ ${ }^{2}$ Universidade Federal do Rio Grande do Norte. Departamento de Química, Natal, Rio Grande do Norte, Brazil \\ *diegofelixdias@gmail.com
}

In this work, FeCo nanoparticles were synthesized in Core-Shell conformation by synthesis called Sol-Gel-Proteic route. This method is derived from the conventional sol-gel, and used as edible gelatin precursor organic. First was prepared a solution containing nitrates of iron and cobalt and gelatin edible, which was under constant agitation so that the mixture became uniformly dispersed, prior to remain in the oven for 48 hours at a temperature of $100{ }^{\circ} \mathrm{C}$. Then, the obtained xerogel was macerate and kept in a rotary kiln for 2 hours at $700{ }^{\circ} \mathrm{C}$ with an air flow of $40 \mathrm{~mL} / \mathrm{min}$ to promote oxidation of the sample. From the oxidation process, the work was divided into two parts. In the first part tried to obtain samples in which the core consisted of the FeCo alloy, while the shell was composed by $\mathrm{Fe}_{3} \mathrm{O}_{4}$. In the second we tried to obtain the opposite structure, with the core formed by the oxide and the shell formed by the alloy. Some conditions were varied during synthesis so as to try to control size of the core and shell, oxidation temperature and observe changes in the magnetization saturation. The samples were characterized by X-ray diffraction, transmission electron microscopy, Thermogravimetry, Vibrant Sample Magnetometry and Mössbauer Spectroscopy, and the results obtained compared with literature studies. 